\title{
WAKEFIELDS IN THE LCLS UNDULATOR TRANSITIONS *
}

\author{
K.L.F. Bane, SLAC, Stanford, CA 94309, USA \\ I. Zagorodnov, DESY, Hamburg, Germany
}

\section{Abstract}

For a short bunch in an elliptical collimator we demonstrate that, as in a purely round collimator, the wake can be estimated from the primary fields of the beam alone. We obtain the wakes in the LCLS rectangular-to-round, undulator transitions using a hybrid method that includes indirect numerical (field) integration and an analytical potential energy term. For the LCLS $1 \mathrm{nC}$ bunch charge configuration, we find the wake-induced energy change in the transitions to be small compared to that due to the resistance of the beam pipe walls.

\section{INTRODUCTION}

In the Linac Coherent Light Source (LCLS) with the nominal, $1 \mathrm{nC}$ of charge configuration the longitudinal wakefields in the undulator region are significant and will affect the laser performance. The dominant contribution to the wakefields is the resistive wall wake (with the ac conductivity included) of the undulator beam pipe [1]. To ameliorate the wake effects it has been proposed to change the pipe inner surface material from copper to aluminum and to change the cross-section from a round to an approximately rectangular shape (while leaving the vertical aperture fixed). Within the undulator, however, the beam pipe will need to be interrupted 33 times by pipes with round cross-sections that house the beam position monitors. Thus there will be 33 pairs of rectangular-to-round transitions. In this note we calculate the (geometric) wakefields generated in these transitions.

To solve this problem by direct (numerical) integration of the fields as the beam moves through the structure is difficult for the short LCLS bunch: to allow the wake to "catch-up" to the beam, integration through a long exit beam pipe is required, a procedure that tends to accumulate numerical errors. An indirect integration algorithm for non-cylindrically symmetric (3D) structures is required. For 3D cavity-like structures with beam pipes, an indirect procedure was derived by $\mathrm{T}$. Weiland and $\mathrm{R}$. Wanzenberg [2]. For cylindrically symmetric (2D), collimatorlike structures, an indirect procedure was developed by O. Napoly, et al; according to this method, after the beam passes the structure, the long on-axis integration of fields is replaced by a radial integration to the walls [3]. In this report we make use of a calculation approach that is applicable to the (3D) LCLS rectangular-to-round transitions.

In an LCLS transition a $10 \mathrm{~mm}$ by $5 \mathrm{~mm}$ (horizontal $x$ by vertical $y$ ) rectangular pipe joins a $4 \mathrm{~mm}$ radius round

\footnotetext{
* Work supported by the Department of Energy, contract DE-AC0276 SF00515 and by the EUROFEL project.
}

pipe (see Fig. 1). The length of a rectangular section is $\sim 3 \mathrm{~m}$, of a round section $\geq 470 \mathrm{~mm}$. Because of the large distance between transitions, a pair of transitions has the same wakefield no matter which transition comes first. In this report numerical calculations are done with $E C H O$, a finite-difference, time-domain computer program that can accurately obtain wakefields of short bunches in long structures (it generates minimal errors due to "mesh dispersion" and mesh-to-boundary mismatch) [4]; the meshing is carried out in Microwave Studio [5]. Note that in the LCLS undulator region the rms bunch length $\sigma_{z}=20 \mu \mathrm{m}$.

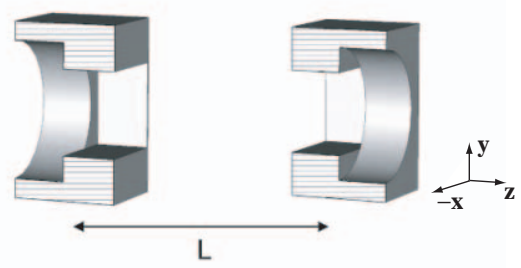

Figure 1: A pair of LCLS rectangular-to-round transitions.

\section{D MODEL}

\section{Analytical Wake}

Let us first estimate the wake potential using a 2D (cylindrically symmetric) model. Consider transitions from a round pipe of radius $a=2.5 \mathrm{~mm}$ to one of radius $b=4 \mathrm{~mm}$ and back again, where the distance between transitions is large. Since the aperture of this 2D model is everywhere less than or equal to that of the real (3D) transition, we expect this estimate of the wake to be pessimistic.

For short bunches $\left(\sigma_{z} \ll a\right)$ the diffraction model of Heifets and Kheifets $(\mathrm{H} \& \mathrm{~K})$ applies [6]: the (high frequency) impedance is constant

$$
Z_{h i}=\frac{Z_{0}}{\pi} \ln (b / a),
$$

with $Z_{0}=377 \Omega$, and the wake is resistive (the wake shape is the same as the bunch distribution)

$$
W(s)=-Z_{h i} c \lambda_{z}(s),
$$

with $s$ position within the bunch, $c$ the speed of light, and $\lambda_{z}$ the bunch distribution (positive $W$ means energy gain). For a Gaussian bunch the loss factor (minus the average wake) is $k_{\text {loss }}=Z_{h i} c /\left(2 \sqrt{\pi} \sigma_{z}\right)$. For our parameters: $Z_{h i}=56.4 \Omega, k_{\text {loss }}=238 \mathrm{~V} / \mathrm{pC}$ and the wake rms $W_{r m s}=94 \mathrm{~V} / \mathrm{pC}$ (in this report we take a $\sigma_{z}=20 \mu \mathrm{m}$ Gaussian bunch unless otherwise indicated).

H\&K explained that the short-bunch wake of an intransition (from big pipe to small) can be written as $W^{i n}=$ 
$W^{r}-W^{e}$ [with $W^{r}, W^{e}$, radiation and (static) potential energy parts], and of an out-transition (from small pipe to big) $W^{\text {out }}=W^{r}+W^{e}$, and that $W^{r} \approx W^{e}$. Thus, for e.g. an in-transition, the two parts cancel to give nearly zero wake (one can think of the fields beyond $a$ as being cleanly clipped away, with the beam never aware of the change in boundary). Thus, for a transition pair $W=2 W^{r} \approx 2 W^{e}$.

\section{Numerical Calculations}

Numerically obtaining the wake for this problem by direct integration of the electric fields as the bunch passes the transitions is difficult, since it takes a long time for the scattered fields to catch up to the bunch. [The catch-up distance $z_{c u} \gtrsim 2(b-a)^{2} / \ell$, with $\ell$ a fraction of $\sigma_{z}$.] A modification of the Napoly indirect method is employed by the computer program $E C H O$ for $2 \mathrm{D}$ calculations [7]. An ECHO calculation was performed for a transition pair of the 2D model; for the calculation the pair was modelled as a collimator of length $g=20 \mathrm{~mm}$; we find that the numerical result agrees well with the analytical approximation (see Fig. 2). Note that numerically we find that the catch-up distance $z_{c u} \approx 2.3 \mathrm{~m}\left(\right.$ or $\left.\ell \approx \sigma_{z} / 10\right)$.

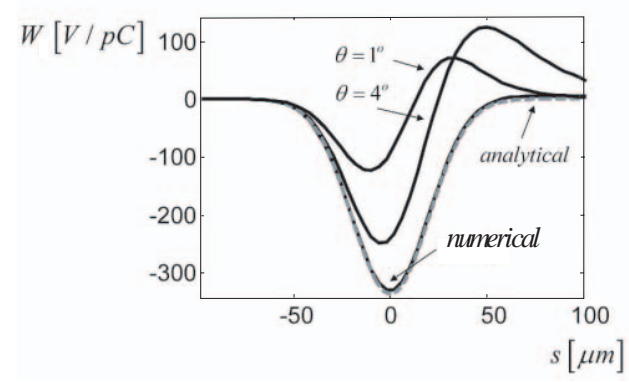

Figure 2: Wake for 2D model of LCLS transition pair (solid), compared with the analytical result, Eq. 2 (dashes). Also given are results for tapered transitions (see below).

Effect of Tapering Diffraction radiation is projected at shallow angles, and one expects tapering to have little effect until taper angle $\theta \sim \sigma_{z} / a$, which here implies $\theta \sim 0.5^{\circ}$. We performed calculations for symmetric $2 \mathrm{D}$ transition pairs with taper angles (see Fig. 3). Wakes, for $\theta=1^{\circ}, 4^{\circ}$, are given in Fig. $2 ; k_{l o s s}$ and $W_{r m s}$, for several angles, are given in Table 1 (the earlier results are labeled $\theta=90^{\circ}$ ). From Table 1 we see that e.g. $W_{r m s}$ does not significantly decrease until $\theta \lesssim 1^{\circ}$, in reasonable agreement with our estimate.

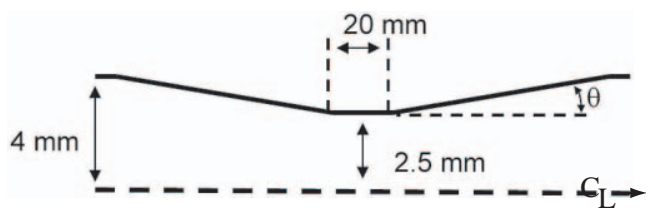

Figure 3: A tapered, cylindrically symmetric collimator.
Table 1: Wake properties for the round collimator of Fig. 3.

\begin{tabular}{|c|c|c|}
\hline$\theta[\mathrm{deg}]$ & $k_{\text {loss }}[\mathrm{V} / \mathrm{pC}]$ & $W_{r m s}[\mathrm{~V} / \mathrm{pC}]$ \\
\hline 90. & 238. & 94. \\
(analytical) & & \\
\hline 90. & 235. & 93. \\
8.5 & 191. & 96. \\
4. & 148. & 101. \\
2. & 88. & 91. \\
1. & 50. & 65. \\
0.5 & 33. & 41. \\
\hline
\end{tabular}

\section{D TRANSITIONS}

\section{Collimator in Round Beam Pipe}

The indirect method has been extended in $E C H O$ to $3 \mathrm{D}$ structures that end in a round beam pipe [8]. For short bunches $E C H O$ employs a moving mesh that encloses the beam longitudinally and reaches transversely to the structure walls. The first part of the calculation is direct integration of the wake forces as the beam passes through the beginning of the structure. When the moving mesh finally is entirely within the round exit pipe region the monopole moment of the transverse field (over the mesh) is extracted; this field is then integrated radially to the wall, following the procedure of the $2 \mathrm{D}$ indirect method.

As a concrete example consider an elliptical collimator in a round beam pipe (see Fig. 4), and a Gaussian bunch with $\sigma_{z}=25 \mu \mathrm{m}$. The wake as obtained by the justdescribed indirect method is shown in Fig. 5 (the solid curve). The loss factor $k_{\text {loss }}=116.6 \mathrm{~V} / \mathrm{pC}$. To obtain an analytical result, we calculate the potential difference in the primary beam field when it is in the elliptical vs. in the round pipe, giving us $W^{e}$ (more details of such a calculation are given in the next section); then, as in the 2D case, we let $W=2 W^{e}$. The result is shown by dashes in Fig. 5; we see good agreement with the numerical result $\left(k_{\text {loss }}=118.5 \mathrm{~V} / \mathrm{pC}\right)$. This agreement suggests that this analytical method can be used to find the short-bunch wakes of a large class of 3D collimators (specifically, those that are abrupt-edged and translationally symmetric).
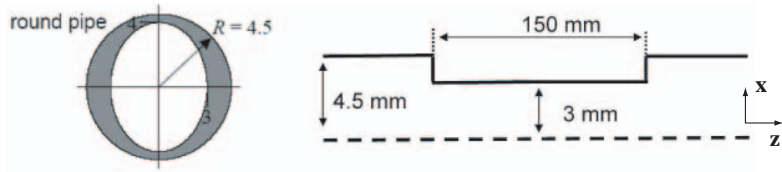

Figure 4: Geometry of 3D collimator in a round beam pipe.

Before leaving this section, recall that in the $2 \mathrm{D}$ (round) collimator case $W^{e}$ could be found either by the change in potential in the primary field in the two pipes, or by the potential in the field "clipped" away by the collimator: the two calculations gave the same answer. For the 3D collimator this is no longer true, because the steady-state field pattern within the (elliptical) collimator aperture differs from that in the (round) beam pipe. Thus, we find here, accord- 


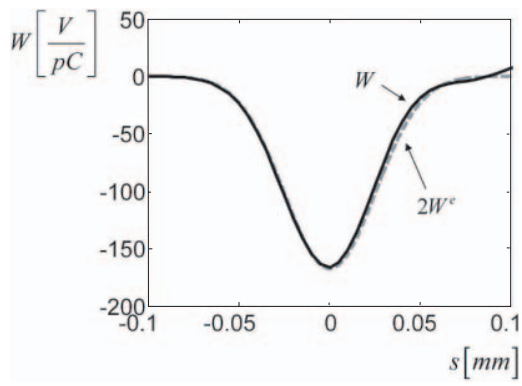

Figure 5: Wake of the elliptical collimator, comparing the numerical (solid line) and analytical results (dashes).

ing to a clipping calculation, $k_{\text {loss }}=110.3 \mathrm{~V} / \mathrm{pC}$; this result is different from before, but it agrees well with a direct numerical calculation for a short ( $3 \mathrm{~mm}$-long) elliptical collimator, where $k_{\text {loss }}=109.9 \mathrm{~V} / \mathrm{pC}$. The implication is that, when a beam encounters a collimator it takes time for its fields to adjust themselves to a new steady-state configuration. Thus, for a short collimator, a clipping calculation is appropriate; for a long collimator the change in potential in the two pipes gives the correct answer.

\section{LCLS Rectangular-to-Round Transitions}

Consider now the pair of LCLS rectangular-to-round transitions of Fig. 1. Both the rectangular and the round regions are long and we can consider them independently. First note that the analytical methods used above for the elliptical collimator-obtaining the wake from the primary field potential alone-cannot be used here, since neither cross-section is inscribed entirely within the other. Also note that the indirect numerical method, described above, can only be applied to the rectangular-to-round half of the pair since it ends in a round pipe. For a transition pair

$$
W=W^{\text {in }}+W^{\text {out }}=2\left(W^{\text {out }}-W^{e}\right),
$$

where $W^{\text {out }}$ represents the numerically-obtained, rectangular-to-round wake and $W^{e}$ the potential difference in primary beam fields in the two pipe cross-sections.

To obtain $W^{e}$ we first use a Matlab Poisson equation solver to find the field energy for the 2D problem of a beam in the rectangular geometry (see Fig. 6). In the round case the normalized energy beyond radius $\epsilon$ is $w=u / Q^{2}=$ $Z_{0} c \ln (b / \epsilon) / 2 \pi$, with $u$ the energy in the (2D) fields, $Q$ the bunch charge. For each geometry the total field energy diverges at the origin, but the difference between the two totals does not (near the origin the fields are the same). We find $\left(w_{\text {round }}-w_{\text {rect }}\right)=0.2407 Z_{0} c / 2 \pi$. Finally, $W^{e}=$ $\lambda_{z}\left(w_{\text {round }}-w_{\text {rect }}\right)$.

To test the indirect method on the LCLS rectangular-toround transitions we first perform calculations for a longer bunch $\left(\sigma_{z}=200 \mu \mathrm{m}\right)$. The calculations are done twice: first, using direct integration on a pair of transitions separated by $L=50 \mathrm{~mm}$, and then using the indirect method. We find good agreement in the results.

For the nominal $20 \mu \mathrm{m}$ long bunch it is difficult to obtain an accurate wake using the direct method. Thus, for this

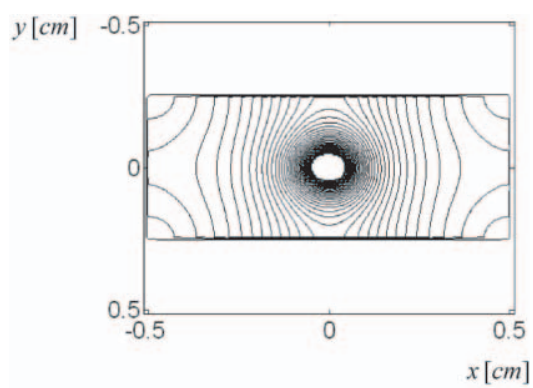

Figure 6: Field energy density in the rectangular beam pipe.

case we perform the indirect calculation only. The results are shown in Fig. 7. We obtain $k_{\text {loss }}=153 \mathrm{~V} / \mathrm{pC}$ and $W_{r m s}=61 \mathrm{~V} / \mathrm{pC}$. Using $Z_{h i}=2 \sqrt{\pi} \sigma_{z} k_{\text {loss }} / c$, we find that $Z_{h i}=36.2 \Omega$. The 3D results are $\sim 2 / 3$ the $2 \mathrm{D}$ estimates (see Table 1 with $\theta=90^{\circ}$ ).

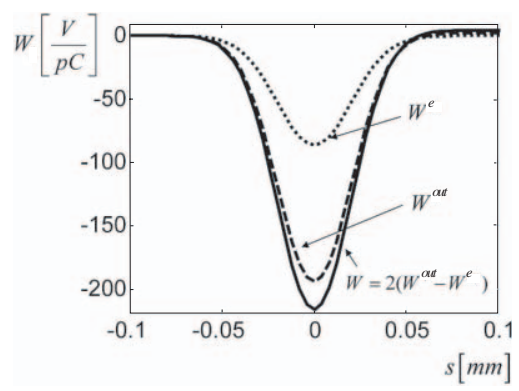

Figure 7: Wake of a pair of LCLS 3D transitions.

The LCLS bunch shape is not Gaussian, and can be described as a $3 \mathrm{kA}$ flat-top with leading and trailing spikes or horns, with an rms length $\sigma_{z}=20 \mu \mathrm{m}$. The total wake is given by Eq. 2, with $Z_{h i}=1.2 \mathrm{k} \Omega$ (the effect of all 33 pairs of rectangular-to-round transitions). It is the variation in induced energy change that is important for the LCLS, since an average change can always be compensated by tapering the undulator strength. Over the core of the beam (that part that excludes the horns) the total variation in induced energy is $\Delta E / E \sim 0.03 \%$ ( $Q=1 \mathrm{nC}$, energy $E=14 \mathrm{GeV})$; in the horn regions it grows to $\Delta E / E \sim 0.10 \%, 0.15 \%$. These numbers, however, are still small compared to the effect of the resistive wall wake of the beam pipe (for the flat, aluminum chamber $\Delta E / E=0.6 \%$ ) [1]

We thank M. Dohlus, S. Heifets, and G. Stupakov for helpful discussions on wakes and impedances, and P. Emma for supplying the LCLS bunch distribution.

\section{REFERENCES}

[1] K.L.F. Bane and G. Stupakov, Proc PAC05, 2005, p. 3390.

[2] T. Weiland and R. Wanzenberg, CCAS-CAT-CERN Accelerator School, 1993, Vol. 1, p. 140.

[3] O. Napoly et al, Num Instrum Meth 344, 255 (1993).

[4] I. Zagorodnov and T. Weiland, PR-STAB 8, 042001 (2005).

[5] CST GmbH, Darmstadt, Germany (www.cst.de).

[6] S. Heifets and S. Kheifets, Rev Mod Phys 63, 631 (1991).

[7] I. Zagorodnov et al, J Comp Phys 191, 525 (2003).

[8] I. Zagorodnov, "Indirect methods for wake potential integration," submitted to PRST-AB (2006). 